Advanced Materials Development and Performance (AMDP2011)

International Journal of Modern Physics: Conference Series

Vol. 6 (2012) 658-663

(c) World Scientific Publishing Company

DOI: $10.1142 / \mathrm{S} 2010194512003935$

\title{
AN EVALUATION ON THE SMART COMPOSITE DAMAGED BY THERMAL SHOCK
}

\author{
JIN KYUNG LEE ${ }^{\dagger}$, SANG PILL LEE ${ }^{1}$ \\ Department of Mechanical Engineering, Dong-Eui University, Busanjin-Gu, Busan, 614-714, Korea \\ leejink@deu.ac.kr ${ }^{\dagger}$, splee87@deu.ac.kr1 \\ YOUNG CHUL PARK ${ }^{2}$ \\ Department of Mechanical Engineering, Dong-A University, Saha-Gu, Busan, 604-714, Korea \\ parkyc67@mail.donga.ac.kr ${ }^{2}$ \\ JOON HYUN LEE 3 \\ School of Mechanical Engineering, Pusan National University, Keumjung-Gu, Busan, 609-735, Korea \\ johlee@pusan.ac.kr
}

\begin{abstract}
A shape memory alloy (SMA) as part of some products and system has been used to keep their shape at any specified temperature. By using this characteristic of the shape memory alloy it can be solved the problem of the residual stress by difference of coefficients of thermal expansion between reinforcement and matrix within composite. In this study, TiNi/A16061 shape memory alloy composite was fabricated through hot press method, and the optimal fabrication condition was created. The bonding effect of the matrix and the reinforcement within the SMA composite was strengthened by cold rolling. The SMA composite can be applied as the part of airplane and vessel, and used under tough condition of repetitive thermal shock cycles of high and low temperatures. Therefore, the thermal shock test was performed for the SMA composite, and mechanical properties were evaluated. The tensile strength of the SMA composite showed a slight decline with the thermal shock cycles. In addition, acoustic emission (AE) technique was used to quantify the microscopic damage behavior of cold rolled TiNi/Al6061 shape memory alloy composite that underwent thermal shock cycles. The damage degree on the specimen that underwent thermal shock cycles was discussed. Actually AE parameters such as AE event, count and energy was analyzed, and these parameters was useful to evaluate the damage behavior and degree of the SMA composite. The waveform of the signal caused by debonding was pulse type, and showed the frequency range of $160 \mathrm{kHz}$, however, the signal by the fiber fracture showed the pulse type of high magnitude and frequency range of $220 \mathrm{kH}$.
\end{abstract}

Keywords: Shape memory alloy, TiNi/Al6061 composite, Hot press, Cold rolling, Acoustic emission technique; AE parameter.

\section{Introduction}

A shape memory alloy (SMA) is a material recovering to the original shape at any temperature even though deformation by external forces. Among the shape memory alloy TiNi system shape memory alloy shows the highest properties for the shape memory 
effect, corrosion resistance and stability ${ }^{(1-3)}$ In this study, shape memory alloy was used to overcome the disadvantages of strength decline by the difference of heat expansion coefficient between reinforcement and matrix in composites, and TiNi/Al6061 shape memory composite was manufactured. TiNi/Al6061 shape memory composite has benefits that it can control the propagation of cracks by shape memory effect as well as strength increase. Due to these merits TiNi/Al6061 shape memory composite is expected as part of aircraft and vessel, and a ship and airplane were often used under severe environment of high and low temperatures. However, many studies for shape memory composite were concentrated upon damage behavior and strength change of the composite at high temperature. As mentioned above, in order to apply it to the various fields of industry lots of researches on characteristics of composite under high and low temperatures should be done, and moreover, study on the influence of thermal shock should be also cleared. Therefore, a study was performed on microscopic damage behavior and strength change of TiNi/Al6061 shape memory composite under low temperature. In addition, damage behavior of the composite caused the cyclic thermal shock was also evaluated. In this study one of nondestructive tests that more effective than failure test for composite evaluation, an acoustic emission (AE) technique was used to clear the damage characteristic. ${ }^{(4-6)}$ The acoustic emission technique has an advantage of evaluation for safety of structure and degree of damage of materials in real time.

\section{Materials and Experiment}

A shape memory alloy in this study is TiNi alloy of $500 \mu \mathrm{m}$ diameters (50-Ti(at\%), $\mathrm{Ms}=303 \mathrm{~K}, \mathrm{As}=327 \mathrm{~K}, \mathrm{Af}=337 \mathrm{~K}, \mathrm{Mf}=290 \mathrm{~K}$ ), and has been arrayed regularly between Al6061 plates. The shape memory composite was manufactured in hot press under the conditions of $803 \mathrm{~K}$ temperature and $50 \mathrm{MPa}$ pressure. The specimen was made in argon atmosphere to prevent the oxidation of $\mathrm{Al} 6061$ material. The debondings were generated at the boundary of the matrix and reinforcement when the specimen that manufactured by this method was loaded. Therefore, the cold rolling was applied to the specimen to strengthen the adhesive strength between the matrix and reinforcement. However, a thermal shock device was designed to evaluate the thermal shock damage of the TiNi/Al6061 composite. Fig. 1 shows the schematic diagram of thermal shock test for the composite. For cyclic thermal shock test the specimen was maintained for 10minutes in the cooling device, and the cooled specimen was preserved for 20 minutes in electric furnace of $303 \mathrm{~K}$ temperature to eliminate the water on the surface of specimen. The dried specimen was dropped into the cooling aparature again. By repetition of these processes the specimen was damaged by thermal shock. The damage evaluation was performed for the specimens that underwent thermal shock damage of 50 cycles, 100 cycles, 300 cycles, 500 cycles and 700 cycles. Tensile test was also conducted to examine the mechanical properties for the damaged specimens. In addition, an acoustic emission technique was applied to measure the microscopic failure characteristics of the specimens that confronted with thermal shock damage. The test was performed at the 


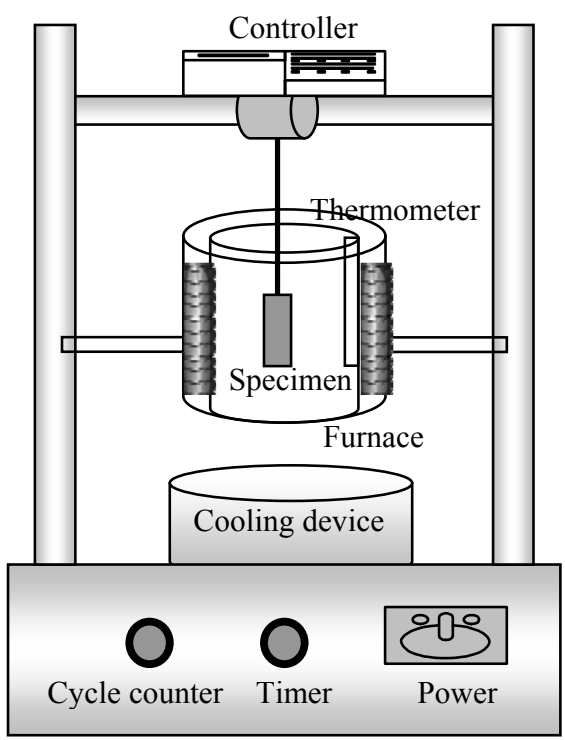

Fig. 1 Schematic diagram of thermal shock test.

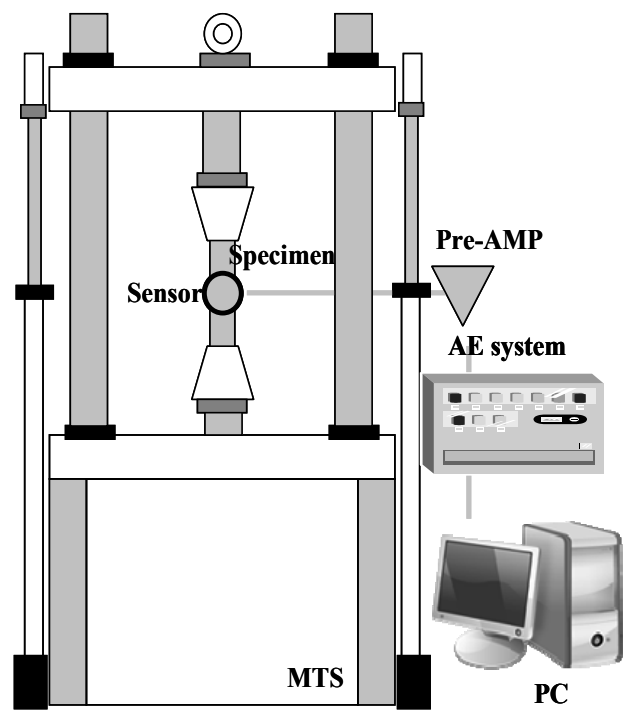

Fig.2 Schematic diagram of tensile strength and AE testes

condition of $1 \mathrm{~mm} / \mathrm{min}$ loading. Fig. 2 shows the schematic diagram of experimental set up for the acoustic emission test. As shown in Fig. 2, lots of elastic waves due to the cracks initiation and propagation were occurred while loading the specimen. The generated elastic waves were detected by the AE sensor attached at the specimen, and amplified by $40 \mathrm{~dB}$ at pre amplifier and analyzed by the parameters at the main board. There are many acoustic emission parameters for evaluation on the degree of the damage of the TiNi/Al6061 and found most useful parameter among AE parameters. The used sensor was wide band $(100 \mathrm{kHz}-1200 \mathrm{kHz})$ and threshold level for elimination of background noise was fixed by $45 \mathrm{~dB}$.

\section{Results and Discussions}

\subsection{Thermal shock characteristics of the composite}

A new thermal shock instrument was designed to evaluate the damage of the shape memory composite by thermal shock. Fig. 3 shows the relationship between the strength and thermal shock cycles. As shown in Fig. 3 the tensile strength and yield strength were reduced linearly with thermal shock cycles. These are because the property of the composite is getting brittle with thermal shock and the cohesion at the boundary of the reinforcement and matrix is getting weak. However, the young's modulus did not change regardless of thermal shock damage. Figures from Fig.4 to Fig.6 show the damage behavior and AE signals for the thermal shock damaged specimen of 500 cycles, and Fig. 4 represented the relationship between the load and AE events. Lots of AE events 
were generated at the elastic stage of the beginning of load. From the beginning of the plastic zone the number of AE events decreased gradually, and showed the tendency of increase again at the $50 \%$ point of the complete breakage. Lots of AE events at the beginning of load were generated due to the debonding of the matrix and reinforcement while tensile loading. In general, AE events were hardly produced at elastic range of metals, whereas a few AE signals can be generated at composite due to the defects such as cracks at the initial stage of the loading. However, in case of the specimen that meet with thermal shock damage of 500 cycles the cohesion between the matrix and reinforcement was getting weak by the thermal shock, and the debonding generated easily and lots of $\mathrm{AE}$ events caused by this phenomenon were produced. The decline of $\mathrm{AE}$ events at the beginning of the plastic zone was caused by a lot of debonding phenomena at the elastic range advance. As the load increased lots of $\mathrm{AE}$ events were generated due to the rapid plastic behavior and debonding. Finally, many AE events were produced due to the fiber's fracture, production of large crack and its growth when complete breakage of the composite. Fig. 5 shows the relationship of AE events and counts. At the beginning of the load, the number of $\mathrm{AE}$ events and counts were increased by steps together, however, the decline stage of $\mathrm{AE}$ events, at the initial plastic range, the number of $\mathrm{AE}$ counts did not almost increase. The number of $\mathrm{AE}$ counts increased abruptly at the $50 \%$ point of the complete breakage like the variation of AE events. The damage degree of the composite can be assumed through the variation of AE counts. Fig. 6 shows the variation of AE energy according to the loading increase. AE signals of the energy below 10 were generated at the elastic zone and at the beginning of the plastic zone, however, many AE signals of the energy above 10 were generated as the plastic stage was processed. This result indicates that the appearance of AE signal above $10 \mathrm{in}$ energy means the arrival of $50 \%$ point of the complete breakage. AE events, counts and energy among AE parameters are useful to evaluate the damage behavior and degree of the composite that underwent thermal shock. Fig. 7 and Fig. 8 show the typical waveform and frequency according to the microscopic damage behavior.

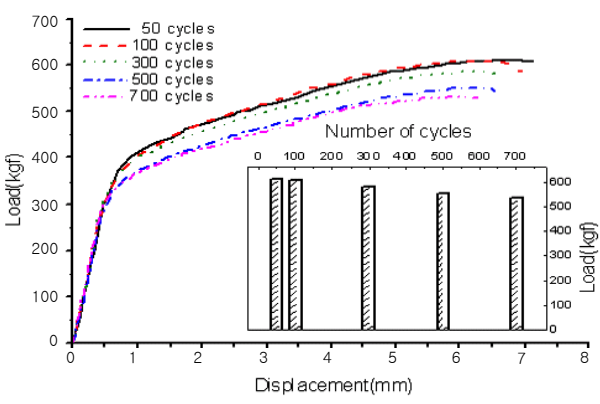

Fig. 3 The strength vs. thermal shock cycles

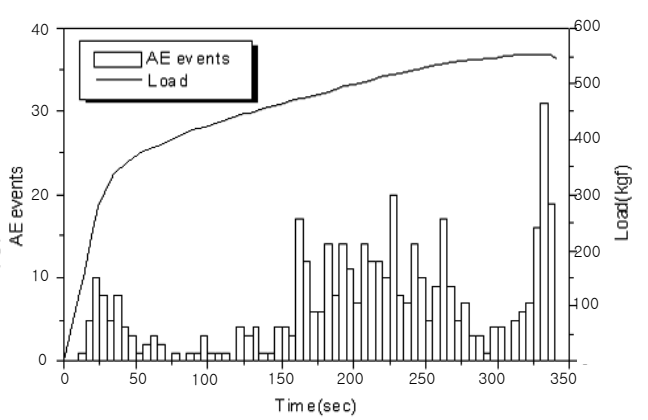

Fig.4 Load vs.AE events 


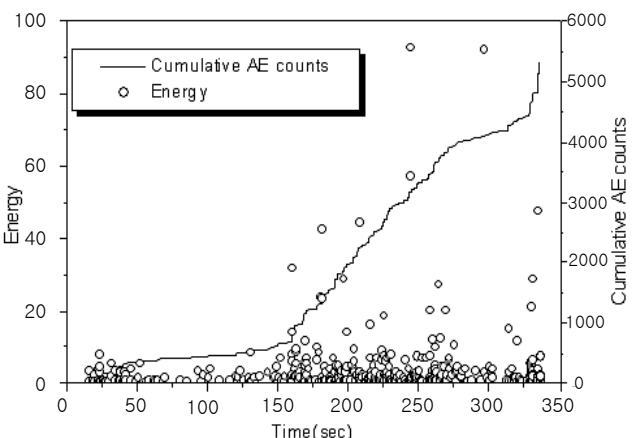

Fig. 5 AE events and counts

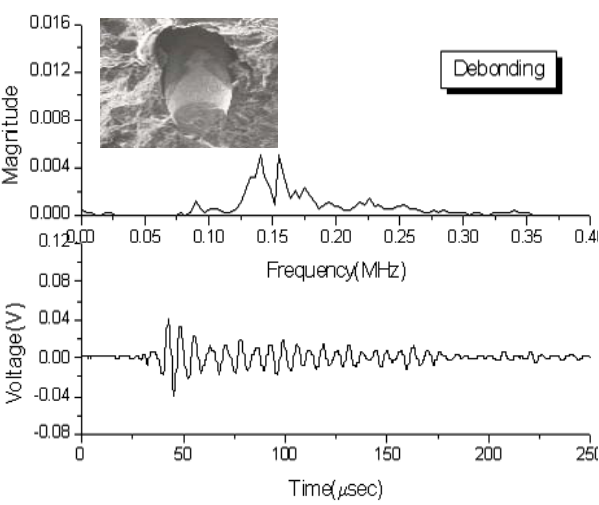

Fig.7 Typical Waveform and frequency range by debonding

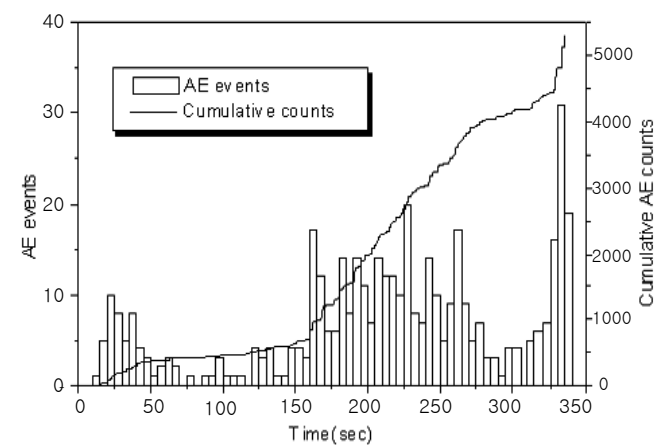

Fig.6 Cumulative AE counts and Energy

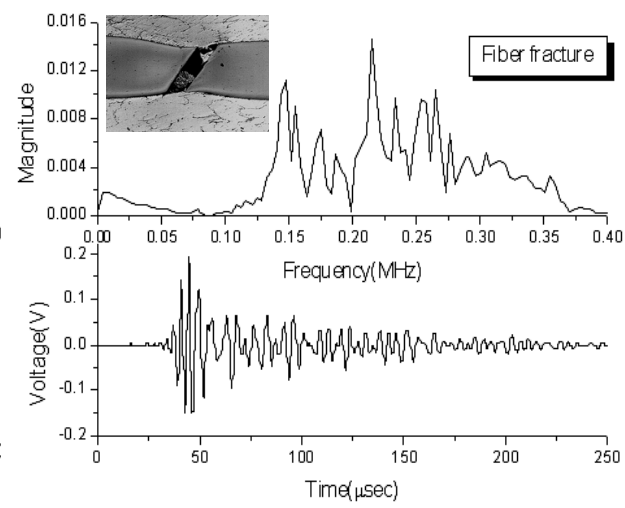

Fig.8 Typical Waveform and frequency range by fiber fracture

Fig. 7 represents the characteristics of waveform and frequency for the signal caused by the debonding at the beginning of the load. The waveform was pulse type, and showed the frequency range of $160 \mathrm{kHz}$. The main frequency range was $160 \mathrm{kHz}$ although the magnitude was changed by the size and direction of the debonding. Fig. 8 shows the waveform and frequency for the signal by the fiber fracture, high magnitude waveform and frequency range of $220 \mathrm{kHz}$. The waveform was similar to one of the debonding, however, the frequency range of the signal by the fiber fracture was far high than that of the signal caused by the debonding.

\section{Conclusions}

From the study on the microscopic damage behavior of the TiNi/Al6061 shape memory composite at low temperature using $\mathrm{AE}$ technique, following results were obtained;

(1) Tensile strength of the composite was decreased as thermal shock cycle increased. 
(2) AE count was a useful parameter to evaluate the damage behavior and the rest life for the composite at low temperature. For the shape memory composite AE event, count and energy was useful to exam the microscopic damage and the degree of damage.

(3) The waveform of the signal caused by debonding was pulse type, and showed the frequency range of $160 \mathrm{kHz}$, however, the signal by the fiber fracture showed the pulse type of high magnitude and frequency range of $220 \mathrm{kHz}$. Both effects are independent and additive.

\section{Acknowledgments}

This work was supported by Korea Institute of Energy Technology Evaluation and Planning (KETEP), through its Technology Innovation Program of Nuclear Power Plant.

\section{References}

1. Yuanti Huang, Gueping Y and, Ping He, Scripta Metallurgica, 19, 1033 (1985).

2. Prahlad, H., and Chopra, I, Smart Structures and Materials 1999: Smart Structures and Integrated Systems, 604 (1999).

3. Arup K., Maji and Ihosvany Negret, J. of Engineering mechanics, 124, 1121 (1998).

4. Bukkapatnam STS., Kumara SRT. and Lakhtakia A, J. of Manufacturing Science \& Engineering, 121, 568 (1999).

5. Okafor, A.C., and Birdsong, S.R, Proc. of the Process Control and Sensors for Manufacturing II, 115 (1999).

6. J.K. Lee, J.H. Lee, H.S. Choi and M.R. Lee, Transactions of the KSME, A, 24, 2520 (2000). 